\title{
A estrutura fatorial do inventário de características da personalidade 1
}

\author{
The factor structure of the personal \\ characteristics inventory
}

Cristiano Mauro Assis GOMES

\begin{abstract}
Resumo
Este artigo discute a natureza valorativa dos traços do modelo big five e investiga a validade do Inventário de Características da Personalidade, analisando sua estrutura fatorial. Fizeram parte da amostra 716 estudantes do ensino fundamental e médio de uma escola particular de Belo Horizonte. Foi empregada a Análise Fatorial Exploratória, com extração pelo método da máxima verossimilhança e rotação Geomin. Foram empregados o alfa de Cronbach e o índice de determinação do fator. Foram estipulados critérios para a eliminação de itens. A solução escolhida entre as diversas obtidas foi aquela com oito fatores $\left(\chi^{2}=345,39, \mathrm{gl}=163\right.$, índice comparativo de ajuste=0,99, raiz quadrada média do erro de aproximação=0,04, raiz quadrática média residual estandartizada=0,02). Os oito traços identificados são: abertura a novas experiências, extroversão, mutabilidade, estabilidade, foco em relações humanas, introversão, foco no objeto e foco no objetivo. O alfa dos fatores variou de 0,59 a 0,80, e a determinação do fator variou de 0,80 a 0,92. Implicações são discutidas.
\end{abstract}

Unitermos: Personalidade. Teoria dos cinco grandes fatores. Teste de validade.

\begin{abstract}
The present paper discusses the evaluative nature of the traits of the big-five model and investigates the validity of the Personal Characteristics Inventory by analysing its factor structure. The sample is provided by 716 primary-and secondary-school students from a private school in Belo Horizonte. An Exploratory Factor Analysis is undertaken with extraction by means of the maximum likelihood method and Geomin rotation. We also used Cronbach's alpha and the factor determination index and criteria were stipulated for the items' elimination. The factor selected from the various solutions obtained was the eight-factor structure $\left(\chi^{2}=345.39, d f=163\right.$, Comparative Fit Index $=0.99$, root mean square error of approximation $=0.04$, standardised root mean square residual $=0.02$ ). The eight traits identified are: openness to new experiences, extroversion, changeability, stability, focus on human relationships, introversion, focus on the object and focus on the goal. The factor alphas ranged from 0.59 to 0.80 and the factor determination index ranged from 0.80 to 0.92. The implications of these results are subsequently discussed.
\end{abstract}

Uniterms: Personality. Five factor personality model. Test validity.

$\boldsymbol{\nabla} \boldsymbol{\nabla} \boldsymbol{\nabla} \boldsymbol{\nabla}$

1 Apoio: Fundação de Apoio à Pesquisa do Estado de Minas Gerais.

2 Universidade Federal de Minas Gerais, Programa de Pós-Graduação em Psicologia, Departamento de Psicologia. Av. Antônio Carlos, 6627, Campus da Pampulha, 31270-901, Belo Horizonte, MG, Brasil.E-mails: <cgomes@fafich.ufmg.br>; <cristianogomes@ufmg.br>.

Agradecimento: ao professor Oto Neri Borges. 
O campo de investigação da personalidade ganhou considerável consistência empírica com o modelo dos cinco grandes fatores, conhecido internacionalmente como big five. Esse modelo foi construído ao longo de décadas de pesquisas e é fruto da articulação entre a agenda lexical e a agenda psicométrica sobre os traços humanos de personalidade. Desse encontro, foram produzidas muitas evidências a respeito da existência de cinco grandes dimensões da personalidade (Costa \& McCrae, 1992).

A maior força do modelo big five é seu fôlego empírico. Vários estudos, em diversos países, apresentam resultados de que os cinco grandes fatores podem ser encontrados em diferentes culturas, etnias e sistemas econômicos. Em função de sua abrangência e solidez, há um incontável número de instrumentos na literatura internacional e nacional que se baseiam no big five (Baker, Victor, Chambers \& Halverson, 2004; Costa \& McCrae, 1992; Goldberg, 1990, 1992; Hutz et al., 1998; Hutz \& Nunes, 2001; John, 1990; McCrae \& John, 1992; Nunes, 2000, 2007; Nunes \& Hutz, 2007; Rabelo et al., 2009).

Apesar da força do modelo, há uma limitação que necessita ser superada. Cada uma das cinco dimensões do big five possui duas polaridades ou traços opostos. As cinco dimensões são extroversão, docilidade, conscienciosidade, abertura e neuroticismo. Todas elas possuem uma polaridade positiva e uma polaridade negativa. A dimensão extroversão possui a polaridade extroversão (nome da dimensão) e a polaridade oposta, introversão. A dimensão docilidade possui a polaridade docilidade (nome da dimensão) e a polaridade oposta, frieza. A dimensão conscienciosidade possui a polaridade conscienciosidade (nome da dimensão) e o polo oposto, inconstância. A dimensão abertura possui a polaridade abertura (nome da dimensão) e a polaridade oposta, obtuso. Por último, a dimensão neuroticismo tem a polaridade neuroticismo (nome da dimensão) e o polo oposto, estabilidade. Usualmente, uma das polaridades de cada dimensão é valorada positivamente, em detrimento da polaridade oposta (traço oposto). Pode-se citar como exemplo que, enquanto o neuroticismo é um traço valorado negativamente, sua polaridade oposta, a estabilidade, é valorada positivamente. $O$ traço neuroticismo é definido usualmente por adjetivos ou frases que expressam características pessoais

210 ou comportamentos disfuncionais, desadaptativos ou psicopatológicos. A estabilidade, polaridade oposta ao neuroticismo, envolve adjetivos ou frases que expressam características pessoais ou comportamentos funcionais e adaptativos de estabilidade emocional.

O problema da valoração pode ser encontrado na maioria dos testes internacionais elaborados para mensurar o big five. O Revised NEO Personality Inventory (R-NEO-PI) aborda os traços neuroticismo, extroversão, abertura a experiência, agradabilidade e conscienciosidade, enfocando positivamente a polaridade presente no nome dessas dimensões, em detrimento do polo oposto presente em cada dimensão. Há uma exceção, o neuroticismo. Seu polo oposto é a polaridade focada positivamente (Costa, McCrae \& Jónsson, 2002). O Five-Factor Personality Inventory mensura os mesmos traços do R-NEO-PI, mas substitui a nomenclatura neuroticismo e abertura a experiência por estabilidade emocional e autonomia, respectivamente. A presença de termos valorativos mantém-se presente nesse instrumento (Hendriks, Hofstee \& Raad, 2002).

O Big Five Questionnaire trata a extroversão como energia e a agradabilidade como amigabilidade, além de mensurar a conscienciosidade, a estabilidade emocional e a abertura. Seus termos são valorativos. Para definir o polo oposto de conscienciosidade, por exemplo, são usadas designações de descuidado, negligente, desorganizado, entre outras (Barbaranelli \& Caprara, 2002). O Hierarchical Personality Inventory for Children mensura conscienciosidade, extroversão, estabilidade emocional, e designa para agradabilidade e abertura os termos benevolência e imaginação, respectivamente. A falta de frases específicas para as polaridades opostas presentes nas dimensões faz com que elas sejam compreendidas apenas como desempenho baixo nos traços medidos (Mervielde \& Fruyt, 2002).

Apesar de ser um instrumento não verbal de medida, o Five-FactorNonverbal Personality Questionnaire apresenta os mesmos problemas de valoração de determinados polos das dimensões (Paunonen \& Ashton, 2002). O Global Personality Inventory é um instrumento voltado especificamente para a medida de traços da personalidade em sua relação com o mundo do trabalho. Ele também valoriza determinadas polaridades em detrimento de outras (Schmit, Kihm \& Robie, 2002). O Traits Personality Questionnaire é baseado no NEO-PI-R, e por isso segue o mesmo padrão desse 
instrumento (Tsaousis, 2002). O Big Five Marker Scales segue a mesma tendência (Perugini \& Di Blas, 2002).

Ao analisar o problema da valoração, pode-se argumentar a favor da definição usual encontrada no big five e a presença valorativa. Por exemplo, é possível argumentar a favor da definição do neuroticismo, a partir do ponto de vista de que ele é um traço da personalidade humana que diferencia pessoas com características emocionais patológicas de pessoas com características emocionais normais. No entanto, o argumento defendido neste trabalho é o de que qualquer polaridade dos traços de personalidade deve ser capaz de indicar tanto características normais como psicopatológicas, se escolhidos adequadamente adjetivos e frases para esse objetivo.

Neste trabalho, defende-se o argumento de que os aspectos psicopatológicos devem ser encontráveis em qualquer polaridade das cinco dimensões do modelo big five. Por exemplo, a extroversão pode apresentar características de autoritarismo e negligência em relação ao "mundo interno". Já a introversão, a polaridade oposta da extroversão, pode apresentar características de isolamento e fobia social.

A partir do argumento apontado, não se justifica investigar as cinco dimensões do big five partindo do postulado apriorístico de que algumas polaridades dos traços sejam mais adequadas do ponto de vista de algum critério de valor, seja ele psicopatológico, sociocultural etc. Buscando contrapor ao postulado a priori de predominância valorativa de uma das polaridades de cada uma das dimensões, foi elaborado o Inventário de Características de Personalidade (ICP) pelo autor deste artigo, em conjunto com alguns colaboradores. OICP tenta excluir de seu conjunto de itens qualquer adjetivo ou frase de valor pejorativo em relação às polaridades de cada um dos cinco grandes fatores. Para isso, o ICP separa cada uma das polaridades das cinco grandes dimensões e visa mensurar dez traços de personalidade, que na realidade são as cinco dimensões do big five com as suas respectivas polaridades separadas. Além da separação das polaridades, o ICP altera a nomenclatura de alguns traços, visando à eliminação da valorização de alguma polaridade em detrimento de outra. Os dez traços visados pelo ICP são: (1) mutabilidade, (2) estabilidade, (3) foco no objeto, (4) foco em relações humanas, (5) foco no objetivo, (6) foco em várias atividades, (7) extroversão, (8) introversão, (9) abertura a novas experiências, e (10) abertura a experiências consolidadas.

As características de cada um dos dez traços de personalidade podem ser brevemente descritas da seguinte maneira:

- Extroversão: indivíduo expansivo, focado em valores externos. Mais ligado ao exterior que ao interior. Comunicativo. Manifesta-se, geralmente, com liberdade e desenvoltura.

- Introversão: indivíduo centrado, voltado para si mesmo. Prefere ficar com poucas pessoas. É seletivo, ligado às experiências íntimas de si mesmo.

- Foco em Relações Humanas: indivíduo ligado ao sentimento e às emoções dos outros. Procura compreender as opiniões alheias e enfatiza as relações interpessoais.

- Foco no Objeto: indivíduo focado na interação com os objetos e não nas relações interpessoais. Os sentimentos e as relações humanas estão subordinados à ação ou à atividade em que o indivíduo está envolvido.

- Foco no Objetivo: possui objetivo definido. Centrado e focado em finalizar o objetivo. Planeja metas e persegue-as até sua conclusão.

- Foco em Várias Atividades: indivíduo que inicia várias tarefas e não se preocupa em concluí-las. Prefere participar de uma variedade de experiências e atividades a focar em um empreendimento até o fim.

- Abertura a Novas Experiências: o indivíduo gosta de procurar caminhos alternativos, pensar em novas formas e maneiras de enfrentar um desafio. Sente prazer em situações novas, que envolvem estratégias ainda não construídas nem consolidadas.

- Abertura a Experiências Consolidadas: o indivíduo prefere relações estáveis e caminhos consolidados. Pouco se aventura por trilhas desconhecidas, preferindo agir por vias já testadas e comprovadamente seguras.

- Mutabilidade: indivíduo sensível a suas emoções. Capaz de vivenciar bruscas mudanças de humor.

- Estabilidade: vivencia poucas variações de humor, apresentando um padrão relativamente constante. 
A nomenclatura dos traços do ICP é determinada em função do argumento já exposto. Os nomes mutabilidade e estabilidade são designados no lugar da expressão "neuroticismo", que indica o aspecto psicopatológico da mutabilidade emocional. O seu polo oposto é a estabilidade emocional.

No lugar do nome "abertura", o ICP designa os traços de abertura a novas experiências e abertura a experiências consolidadas. A palavra "abertura" e sua caracterização tradicional tende a indicar um valor pejorativo a seu polo oposto, sugerindo que este apresenta um pensamento obtuso e pouco aberto. Nesse sentido, o nome "abertura a experiências consolidadas" é usado no ICP, ao indicar que pessoas com ênfase nesse polo são aquelas abertas a situações onde há um caminho bem estabelecido e com um histórico pregresso de estratégias bem sucedidas.

Foco no objetivo e foco em várias atividades fazem parte do ICP, no lugar de conscienciosidade. Por sua vez, foco em relações humanas e foco no objeto são usados no ICP no lugar de agradabilidade.

Para a construção do ICP foi feita uma análise dos adjetivos presentes nos principais dicionários da língua portuguesa. Obteve-se, inicialmente, um conjunto de 869 adjetivos. Após uma filtragem inicial, permaneceram 294 adjetivos. Eles foram agrupados dentro do modelo postulado dos dez traços do ICP. Uma lista com os 294 adjetivos foi repassada a dez juízes com a instrução de alocar cada adjetivo em um traço. Teve-se o cuidado de nomear e descrever os traços, de forma que não mobilizassem nenhuma carga valorativa em sua categorização. Após a classificação dos adjetivos pelos juízes, 209 foram considerados adequados diante do critério de bom descritor do traço.

Um piloto foi realizado com oito estudantes do ensino fundamental e ensino médio, buscando verificar quais adjetivos apresentavam significado difícil e deveriam ser eliminados. Foram mantidos 92 adjetivos. Uma nova análise entre juízes foi realizada, sendo selecionados somente os adjetivos que se enquadraram nos dez traços e que foram caracterizados como ótimos pelos juízes, resultando em um total de 50 adjetivos, cinco para cada traço. Uma nova análise foi realizada, visando eliminar qualquer conotação valorativa. Essa análise gerou a eliminação de alguns adjetivos, que

212 foram substituídos por frases, gerando a versão do instrumento que é analisada neste trabalho (Pinheiro, Gomes \& Braga, 2009).

Tendo em vista que o ICP traz uma abordagem inovadora de investigação dentro do campo de estudos sobre o big five, mas carece de estudos sobre sua validade de construto, este artigo investiga em termos exploratórios a validade do ICP, analisando especificamente sua estrutura fatorial. Pretende-se verificar quantas polaridades opostas o ICP é capaz de mensurar, em termos de traços distintos, em uma amostra de estudantes do ensino fundamental e médio. As implicações dos resultados e as limitações do estudo são relatadas e discutidas.

\section{Método}

\section{Participantes}

Fizeram parte da amostra 716 participantes do ensino fundamental e médio de uma escola particular de Belo Horizonte (MG), sendo 379 (53,0\%) do sexo feminino e 337 (47,0\%) do sexo masculino, com idade variando entre 8 e 18 anos $(M=13,75$ e $D P=2,11)$. Do total de participantes, 59,0\% eram oriundos do ensino fundamental, cuja distribuição se deu na seguinte frequência: 94 alunos da sexta série (13,1\%); 110 alunos da sétima série (15,3\%); 99 alunos da oitava série (13,8\%) e 119 alunos da nona série (16,2\%). Já no ensino médio, a distribuição por séries foi de 97 alunos da primeira série (13,5\%); 100 alunos da segunda série (13,9\%) e 97 alunos da terceira série (13,5\%). Já no ensino fundamental, a distribuição deu-se na seguinte frequência: 94 alunos da sexta série (13,1\%); 110 alunos da sétima série (15,3\%); 99 alunos da oitava série (13,8\%) e 119 alunos da nona série (16,6\%).

\section{Instrumentos}

Composto por 50 adjetivos/frases, o ICP tem como objetivo verificar características e ações que indicam dez traços da personalidade. Em nível teórico, os itens são agrupados dentro de cada traço.

O ICP é um instrumento de autorrelato. Sua aplicação depende de um nível mínimo de escolarização, correspondente à sexta série do ensino fundamental, podendo ser feita de forma individual ou coletiva. Não 
há tempo-limite de aplicação. Para cada adjetivo ou frase contidos no item, o respondente deve selecionar uma das cinco opções de uma escala tipo-Likert: (1) nem um pouco, (2) um pouco, (3) mais ou menos, (4) muito, (5) totalmente.

A aplicação do instrumento foi realizada no início de 2008, em 19 turmas de uma escola particular de Belo Horizonte, sendo 13 do ensino fundamental e 6 do ensino médio. O ICP foi aplicado de forma coletiva e sempre por psicólogos ou estudantes de Psicologia devidamente treinados. Foram tomados todos os cuidados éticos em relação aos participantes. A pesquisa contou com a aprovação do Comitê de Ética em Pesquisa da Universidade Federal de Minas Gerais, sob protocolo no 456/07, de 12 de novembro de 2007, tendo todos os participantes assinado o Termo de Consentimento livre e esclarecido antes de sua inclusão na amostra.

Para analisar a estrutura fatorial do ICP, foi empregada a Análise Fatorial Exploratória (AFE), com extração pelo método da máxima verossimilhança, rotação geomin. Todas as AFE utilizaram matrizes de correlação policóricas dos dados. O software estatístico empregado foi o Mplus 5.2. Visando verificar a confiabilidade dos fatores obtidos, foram empregados o alfa de Cronbach e o índice de determinação do fator, disponibilizado pelo Mplus 5.2.

Duas estratégias foram realizadas para análise da estrutura fatorial. A primeira delas consistiu no uso da AFE para grupos de itens. Cada grupo foi criado em função de seu pertencimento às dimensões do modelo big five. Os itens relacionados aos traços mutabilidade e estabilidade compuseram o grupo um. Os itens dos traços foco no objetivo e foco em atividades variadas compuseram o grupo dois. Os itens pertencentes aos traços extroversão e introversão compuseram o grupo três. Os itens relacionados aos traços foco em relações humanas e foco no objeto compuseram o grupo quatro, e os itens relacionados aos traços abertura a experiências novas e abertura a experiências consolidadas compuseram o grupo cinco. O objetivo dessa análise foi verificar a adequação dos itens para mensurar os traços específicos do instrumento.

A análise fatorial exploratória de cada um dos grupos de itens deveria indicar a presença de apenas dois fatores. Itens que mobilizassem a presença de mais do que dois fatores, ou não carregassem de forma satisfatória (carga fatorial igual ou superior a 0,50) o fator esperado, eram eliminados nessa etapa de análise. A segunda estratégia de análise empregada foi a elaboração de uma segunda AFE, com todos os itens selecionados após a primeira estratégia.

Para determinar quantos fatores deveriam ser retidos nas AFE, foram empregados três índices estatísticos: o índice comparativo de ajuste - CFI (comparative fit index) (Bentler, 1990); a estatística raiz quadrada média do erro de aproximação Root Mean Square Error of Approximation (RMSEA); e estatística raiz quadrática média Residual Estandartizada Standardized Root Mean Square Residual(SRMR). A solução fatorial exploratória, com seu respectivo número de fatores retidos, deveria possuir CFI $\geq 0,95$ (Hu \& Bentler, 1999) RMSEA $\leq 0,05$ (Browne \& Cudeck,1993) e SRMR $\leq 0,05$ (Byrne, 2001). Quanto ao uso dos indicadores CFI, REMSEA e SRMR em soluções exploratórias neste trabalho, é reconhecido que eles são usualmente encontrados em análises fatoriais confirmatórias. A retenção de fatores é usualmente feita por meio do critério do autovalor-maior-do-que-um, do teste do scree plot ou da análise paralela. Por sua vez, nenhum desses critérios é amplamente confiável. No entanto, o software Mplus 5.2, de Muthén \& Muthén, disponibiliza os indicadores CFI, RMSEA e SRMR para a verificação do ajuste de soluções exploratórias, o que permite ao pesquisador usar esses índices em AFE, trazendo maior confiabilidade à seleção dos fatores retidos e à escolha da solução exploratória obtida.

\section{Resultados e Discussão}

A análise dos grupos de itens relacionados às dimensões do big five e suas polaridades indicou que vários itens deveriam ser eliminados. Algumas soluções indicavam a presença de três fatores devido à inadequação de itens dentro do grupo. Foram realizadas várias análises até que a solução fatorial de cada grupo apresentasse apenas dois fatores, com boas cargas fatoriais. Em dois casos não foi possível obter dois fatores em um grupo, mas apenas um fator. Esse foi o caso do grupo da abertura a novas experiências e abertura a experiências consolidadas, e do grupo do foco no objetivo e foco em várias atividades. Itens do último traço desses dois grupos tiveram de ser eliminados, de forma que apenas o primeiro traço de cada um desses grupos pôde ser mensurado. 
Dentre os 50 itens, 23 foram excluídos, de modo que 27 itens foram mantidos para a análise fatorial de todos os itens do instrumento. Dentre as diversas soluções obtidas com os 27 itens, foi escolhida aquela com oito fatores $\left(\chi^{2}=345,39, \mathrm{gl}=163, \mathrm{CFI}=0,99\right.$, $\operatorname{RMSEA}=0,04, \mathrm{SRMR}=0,02)$. Os autovalores da matriz policórica analisada são apresentados na Tabela 1. Conforme pode ser observado nessa Tabela, os oito fatores do modelo explicam 65,30\% da variância comum dos itens do instrumento.

A interpretação do significado de cada fator foi feita através da análise dos itens que apresentavam carga fatorial igual ou superior a 0,30 no fator, partindo-se dos itens com maior carga fatorial. Os oito fatores correspondem a oito traços postulados pelo ICP. São eles (Tabela 2): Abertura a Novas Experiências (ANE), Extroversão (Ext), Mutabilidade (Mut), Estabilidade (Est), Foco em Relações Humanas (FRH), Introversão (Int), Foco no Objeto (FO), e Foco no Objetivo (FObj).

A confiabilidade de cada fator foi verificada através do índice de determinação do fator (factor

Tabela 1. Autovalores obtidos da matriz policórica de 27 itens.

\begin{tabular}{|c|c|c|c|c|}
\hline & Autovalores & Escores & Comunalidade \% & Total explicado \% \\
\hline & 1 & 5,06 & 18,76 & 65,30 \\
\hline & 2 & 4,07 & 15,09 & \\
\hline & 3 & 2,80 & 10,37 & \\
\hline & 4 & 1,49 & 5,51 & \\
\hline & 5 & 1,21 & 4,47 & \\
\hline & 6 & 1,04 & 3,84 & \\
\hline & 7 & 1,02 & 3,79 & \\
\hline & 8 & 0,94 & 3,47 & \\
\hline & 9 & 0,81 & & \\
\hline & 10 & 0,72 & & \\
\hline & 11 & 0,67 & & \\
\hline & 12 & 0,61 & & \\
\hline & 13 & 0,60 & & \\
\hline & 14 & 0,56 & & \\
\hline & 15 & 0,55 & & \\
\hline & 16 & 0,53 & & \\
\hline & 17 & 0,53 & & \\
\hline \multirow{7}{*}{ 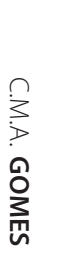 } & 18 & 0,50 & & \\
\hline & 19 & 0,49 & & \\
\hline & 20 & 0,43 & & \\
\hline & 21 & 0,40 & & \\
\hline & 22 & 0,39 & & \\
\hline & 23 & 0,35 & & \\
\hline & 24 & 0,35 & & \\
\hline \multirow[t]{2}{*}{ 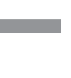 } & 25 & 0,33 & & \\
\hline & 26 & 0,29 & & \\
\hline 4 & 27 & 0,27 & & \\
\hline
\end{tabular}

determinacies), disponível no software Mplus 5.2. Esse índice disponibiliza um valor que indica o grau de confiabilidade do escore fatorial gerado a partir da identificação do fator. O ponto de corte do valor adequado para a determinação do fator é razoavelmente semelhante ao alfa de Cronbach. Valores acima de 0,70 podem ser considerados satisfatórios, e valores acima de 0,90 são considerados ideais. Todos os fatores identificados apresentaram determinação do fator acima de 0,80, de modo que se pode considerar satisfatória a confiabilidade dos seus escores fatoriais (Tabela 2).

Além da análise dos escores fatoriais, também foi verificada a consistência interna dos itens de cada fator, através do alfa de Cronbach. Ambos os critérios foram utilizados neste estudo como indicadores da confiabilidade dos fatores. Conforme pode ser observado na Tabela 2, os alfas variaram de 0,59 a 0,80. Considerando como critério aceitável de consistência interna o valor igual ou superior a 0,70, quatro dos oito fatores atingiram ou ultrapassaram esse ponto de corte. Estabilidade $(0,67)$, foco em relações humanas $(0,64)$, foco no objetivo $(0,62)$ e foco no objeto $(0,59)$ mostraram um alfa inferior a 0,70, demandando maior quantidade de itens para que seus alfas aumentem e cresça a confiabilidade de suas medidas.

Analisando-se a estrutura fatorial de oito fatores, conjuntamente à constatação de que os fatores com baixo alfa são carregados por poucos itens, poder-se-ia argumentar sobre a necessidade de rever a estrutura fatorial e a quantidade obtida de fatores. Uma solução com menor número de fatores poderia indicar traços mais confiáveis, com maior número de itens em cada fator, um maior alfa e medidas mais satisfatórias. De fato, essa estratégia tem uma fundamentação adequada para a obtenção da melhor medida possível com os itens atuais. No entanto, ao investigar a estrutura fatorial do instrumento analisado, busca-se também fomentar a melhoria do mesmo, de forma a ampliar a possibilidade da medida dos polos opostos do big five. A manutenção dos traços com menor alfa é uma escolha teórica e uma aposta futura de que a aquisição de novos itens possibilitará uma medida adequada dos mesmos, assim como a geração de um instrumento inovador, capaz de mensurar separadamente as dez polaridades do big five, que tradicionalmente têm sido mensuradas de forma aglutinada, não distinta, e com um viés valorativo. 
Tabela 2. Solução fatorial obtida: cargas e fatores.

\begin{tabular}{|c|c|c|c|c|c|c|c|c|c|}
\hline Itens/fatores & & 1 & 2 & 3 & 4 & 5 & 6 & 7 & 8 \\
\hline & & ANE & Ext & Mut & Est & $\mathrm{FRH}$ & Int & FO & FObj \\
\hline Sintonizado com várias emoções & $\mathrm{i} 02$ & 0,09 & 0,05 & 0,61 & 0,04 & $-0,17$ & 0,12 & $-0,13$ & 0,08 \\
\hline Estável & $\mathrm{i} 04$ & 0,01 & 0,00 & $-0,10$ & 0,62 & $-0,02$ & 0,06 & 0,00 & 0,01 \\
\hline Falador & 106 & 0,03 & 0,63 & 0,06 & $-0,06$ & $-0,27$ & $-0,04$ & $-0,03$ & $-0,05$ \\
\hline Reservado & i07 & 0,02 & $-0,03$ & 0,07 & 0,06 & 0,10 & 0,67 & 0,04 & $-0,01$ \\
\hline Inventivo & $\mathrm{i} 08$ & 0,92 & $-0,03$ & 0,01 & $-0,01$ & $-0,04$ & 0,04 & 0,01 & $-0,03$ \\
\hline Comunicativo & 109 & 0,02 & 0,71 & 0,02 & $-0,01$ & $-0,01$ & $-0,10$ & 0,03 & 0,14 \\
\hline Influenciado pelas emoções & $\mathrm{i} 10$ & $-0,02$ & 0,05 & 0,75 & $-0,13$ & $-0,08$ & 0,11 & 0,04 & 0,06 \\
\hline Focado & i16 & 0,03 & 0,07 & 0,02 & 0,09 & 0,21 & 0,06 & $-0,01$ & 0,44 \\
\hline Prestativo & i17 & 0,05 & 0,37 & $-0,08$ & 0,00 & 0,58 & 0,05 & $-0,02$ & 0,11 \\
\hline Não emotivo & ¡18 & 0,01 & 0,13 & $-0,39$ & 0,17 & 0,01 & 0,15 & 0,36 & $-0,07$ \\
\hline Criativo & $\mathrm{i} 21$ & 0,61 & 0,13 & $-0,01$ & 0,00 & 0,11 & $-0,11$ & 0,00 & 0,05 \\
\hline Não gosta de mostrar muito afeto & $\mathrm{i} 24$ & 0,06 & 0,01 & 0,01 & 0,01 & 0,06 & 0,19 & 0,63 & 0,02 \\
\hline Gentil & $i 25$ & $-0,02$ & 0,03 & 0,23 & 0,38 & 0,43 & $-0,19$ & 0,05 & $-0,04$ \\
\hline Equilibrado & i27 & 0,08 & $-0,07$ & $-0,02$ & 0,59 & 0,19 & $-0,01$ & $-0,01$ & 0,00 \\
\hline Extrovertido & $\mathrm{i} 29$ & 0,02 & 0,54 & 0,21 & 0,10 & 0,01 & $-0,15$ & 0,03 & $-0,04$ \\
\hline Cooperativo & $i 30$ & $-0,01$ & 0,39 & 0,03 & 0,01 & 0,61 & 0,06 & $-0,15$ & 0,00 \\
\hline Afetivo & $i 32$ & 0,03 & 0,08 & 0,26 & 0,19 & 0,05 & 0,02 & $-0,61$ & $-0,01$ \\
\hline Age com os sentimentos & $i 34$ & $-0,04$ & 0,01 & 0,76 & 0,04 & 0,06 & $-0,04$ & $-0,03$ & $-0,06$ \\
\hline Bom de papo & i37 & $-0,02$ & 0,63 & $-0,02$ & 0,05 & 0,06 & $-0,07$ & 0,01 & 0,09 \\
\hline Autodisciplinado & i39 & 0,05 & $-0,20$ & 0,13 & 0,26 & 0,30 & $-0,06$ & 0,05 & 0,31 \\
\hline Tranquilo & $i 40$ & $-0,04$ & $-0,16$ & 0,00 & 0,53 & 0,16 & 0,08 & $-0,02$ & 0,07 \\
\hline Decidido & $i 41$ & $-0,01$ & 0,12 & 0,00 & 0,34 & $-0,02$ & $-0,08$ & 0,00 & 0,42 \\
\hline Constante & $i 42$ & $-0,02$ & 0,07 & $-0,04$ & 0,57 & $-0,16$ & 0,04 & $-0,04$ & 0,21 \\
\hline Emocional & $i 46$ & 0,00 & $-0,06$ & 0,73 & $-0,08$ & 0,07 & 0,00 & $-0,17$ & $-0,04$ \\
\hline Gosta de cumprir metas & $i 47$ & $-0,01$ & $-0,04$ & 0,01 & $-0,04$ & 0,19 & 0,02 & $-0,03$ & 0,57 \\
\hline Discreto & $i 49$ & 0,00 & $-0,02$ & $-0,09$ & $-0,02$ & 0,30 & 0,57 & $-0,03$ & 0,06 \\
\hline Fechado & $i 50$ & $-0,04$ & $-0,11$ & 0,03 & 0,00 & $-0,03$ & 0,74 & 0,07 & $-0,08$ \\
\hline Determinação do fator & & 0,92 & 0,91 & 0,92 & 0,88 & 0,87 & 0,89 & 0,83 & 0,80 \\
\hline alfa & & 0,70 & 0,75 & 0,80 & 0,67 & 0,64 & 0,70 & 0,59 & 0,62 \\
\hline
\end{tabular}

i: item; ANE: abertura a novas experiências; Ext: Extroversão; Mut: Mutabilidade; Est: Estabilidade; FRH: Foco em Relações Humanas; Int: Introversão; FO: Foco no Objeto; FObj: Foco no Objetivo. Os números tarjados representam as cargas salientes dos itens.

Do ponto de vista da distribuição de respostas dos participantes, o item quatro, "estável", é aquele que apresenta a distribuição mais próxima da curva normal. Conforme pode ser observado na Tabela 4, a maioria das respostas dos participantes nesse item concentrouse na opção central da escala (categoria 3 - c3), com $43 \%$ das respostas. As categorias um e cinco (c1 e c5) representam as extremidades da escala tipo-Likert do ICP e foram respondidas, respectivamente, por 10\% e $7 \%$ dos participantes. As categorias dois e quatro (c2 e c4) representam pontos de dispersão mais próximos ao ponto central da escala tipo-Likert do ICP e foram respondidas, respectivamente, por 18\% e 22\% dos participantes. A média das respostas nesse item foi de 2,99, muito próxima do ponto central da escala (categoria 3 - c3), e desvio-padrão de 1,03.
Através da análise da média de respostas dos participantes nos itens, foi utilizado um ponto de corte arbitrário para classificação do grau de dificuldade dos itens. Esse ponto de corte foi definido arbitrariamente pelo pesquisador, através da análise da distribuição das respostas dos participantes nas categorias da escala tipo Likert do ICP.

Foram formados dois grupos de itens: equilibrados e desequilibrados. Eles foram definidos por meio da elaboração de um Índice de Desequilíbrio (ID). Para cada item, foi feita uma soma da frequência das respostas dos participantes nos pontos um e dois da escala tipo Likert do ICP. Os pontos um e dois da escala indicam que a característica representada pelo item é pouco frequente. Em seguida, para cada item foi somada a frequência de respostas dos participantes nos pontos 
Tabela 3. Estatística descritiva do inventário de características de personalidade.

\begin{tabular}{|c|c|c|c|c|c|c|c|c|c|}
\hline & & $\mathrm{C} 1 \%$ & $\mathrm{C} 2 \%$ & $\mathrm{C} 3 \%$ & $\mathrm{C} 4 \%$ & $\mathrm{C} 5 \%$ & M & $\mathrm{DP}$ & ID \\
\hline Sintonizado com várias emoções & $\mathrm{i} 02$ & 03 & 10 & 33 & 33 & 21 & 3,60 & 1,01 & 4,29 \\
\hline Estável & i04 & 10 & 18 & 43 & 22 & 07 & 2,99 & 1,03 & 1,06 \\
\hline Falador & i06 & 07 & 13 & 31 & 25 & 24 & 3,46 & 1,18 & 2,49 \\
\hline Reservado & i07 & 16 & 33 & 30 & 16 & 05 & 2,63 & 1,09 & 2,25 \\
\hline Inventivo & i08 & 08 & 23 & 29 & 24 & 16 & 3,17 & 1,18 & 1,29 \\
\hline Comunicativo & i09 & 02 & 09 & 26 & 34 & 29 & 3,79 & 1,02 & 5,75 \\
\hline Influenciado pelas emoções & $\mathrm{i} 10$ & 09 & 23 & 29 & 24 & 15 & 3,15 & 1,19 & 1,26 \\
\hline Focado & $¡ 16$ & 04 & 12 & 35 & 31 & 17 & 3,44 & 1,05 & 2,87 \\
\hline Prestativo & ¡17 & 02 & 06 & 26 & 40 & 26 & 3,84 & 0,94 & 8,97 \\
\hline Não emotivo & $¡ 18$ & 49 & 22 & 20 & 07 & 03 & 1,92 & 1,10 & 7,46 \\
\hline Criativo & $\mathrm{i} 21$ & 03 & 10 & 26 & 33 & 29 & 3,75 & 1,06 & 5,01 \\
\hline Não gosta de mostrar muito afeto & $\mathrm{i} 24$ & 36 & 24 & 27 & 08 & 05 & 2,22 & 1,16 & 4,58 \\
\hline Gentil & $\mathrm{i} 25$ & 01 & 02 & 17 & 49 & 31 & 4,08 & 0,79 & 27,72 \\
\hline Equilibrado & $\mathrm{i} 27$ & 03 & 10 & 40 & 33 & 14 & 3,44 & 0,96 & 3,53 \\
\hline Extrovertido & $\mathrm{i} 29$ & 03 & 07 & 22 & 33 & 35 & 3,90 & 1,06 & 6,97 \\
\hline Cooperativo & $\mathrm{i} 30$ & 01 & 06 & 23 & 48 & 22 & 3,84 & 0,86 & 10,45 \\
\hline Afetivo & $\mathrm{i} 32$ & 02 & 07 & 25 & 39 & 28 & 3,84 & 0,97 & 7,58 \\
\hline Age com os sentimentos & ¡34 & 06 & 21 & 30 & 25 & 19 & 3,31 & 1,16 & 1,67 \\
\hline Bom de papo & $i 37$ & 03 & 06 & 27 & 37 & 27 & 3,80 & 1,00 & 7,45 \\
\hline Autodisciplinado & i39 & 03 & 13 & 37 & 30 & 17 & 3,45 & 1,03 & 2,95 \\
\hline Tranquilo & $i 40$ & 06 & 11 & 32 & 31 & 21 & 3,49 & 1,12 & 3,02 \\
\hline Decidido & $i 41$ & 04 & 08 & 23 & 35 & 31 & 3,81 & 1,07 & 5,63 \\
\hline Constante & $i 42$ & 07 & 17 & 46 & 22 & 08 & 3,07 & 1,00 & 1,25 \\
\hline Emocional & $i 46$ & 06 & 19 & 29 & 26 & 20 & 3,36 & 1,17 & 1,85 \\
\hline Gosta de cumprir metas & $i 47$ & 04 & 09 & 23 & 30 & 35 & 3,82 & 1,12 & 4,98 \\
\hline Discreto & $\mathrm{i} 49$ & 15 & 26 & 34 & 18 & 08 & 2,77 & 1,13 & 1,60 \\
\hline Fechado & i50 & 43 & 29 & 17 & 06 & 05 & 2,01 & 1,13 & 6,54 \\
\hline
\end{tabular}

\%: porcentagem; i: Item; C: Categoria; M: Média; DP: Desvio-Padrão; Id: Indice de Desequilíbrio.

Tabela 4. Autovalores obtidos da matriz policórica de 50 itens.

\begin{tabular}{cccccccccc}
\hline Av & Escore & Av & Escore & Av & Escore & Av & Escore & Av & Escore \\
\hline 1 & 7,08 & 11 & 1,12 & 21 & 0,75 & 31 & 0,53 & 41 & 0,37 \\
2 & 5,61 & 12 & 1,06 & 22 & 0,74 & 32 & 0,52 & 42 & 0,34 \\
3 & 3,23 & 13 & 1,05 & 23 & 0,70 & 33 & 0,50 & 43 & 0,33 \\
4 & 2,23 & 14 & 0,96 & 24 & 0,68 & 34 & 0,48 & 44 & 0,32 \\
5 & 1,73 & 15 & 0,95 & 25 & 0,67 & 35 & 0,47 & 45 & 0,30 \\
6 & 1,58 & 16 & 0,93 & 26 & 0,63 & 36 & 0,46 & 46 & 0,27 \\
7 & 1,56 & 17 & 0,89 & 27 & 0,63 & 37 & 0,43 & 47 & 0,27 \\
8 & 1,23 & 18 & 0,84 & 28 & 0,59 & 38 & 0,41 & 48 & 0,26 \\
9 & 1,22 & 19 & 0,83 & 29 & 0,58 & 39 & 0,40 & 49 & 0,21 \\
10 & 1,15 & 20 & 0,78 & 30 & 0,57 & 40 & 0,37 & 50 & 0,19 \\
\hline
\end{tabular}

Av: Autovalores.

quatro e cinco da mesma escala. Os pontos quatro e cinco indicam que a característica representada pelo item é muito frequente. Na medida em que o ponto três da escala é neutro, ele não entrou no cálculo. Em seguida, para cada item foi observado quem possuía a maior frequência de respostas: o grupo dos pontos um e dois ou o grupo dos pontos quatro e cinco. O valor do grupo de maior frequência era dividido, então, pelo valor do grupo de menor frequência. Se o valor do ID não ultrapassasse 1,60, o item poderia ser considerado equilibrado, pois apresentava uma distribuição razoavelmente semelhante a uma curva normal, onde as categorias centrais eram mais frequentemente respondidas pelos participantes do que os extremos da escala. Itens com ID superior a 1,60 apresentavam um desequilíbrio de respostas que pendia ora para o grupo de pontos um e dois da escala, ora para o grupo quatro e cinco.

Através desse critério, pôde-se observar na Tabela 3 que cinco itens do ICP são equilibrados. São eles: estável, inventivo, influenciado pelas emoções, constante e discreto. O restante dos itens pende para o grupo de pontos um e dois ou para o grupo de pontos quatro e cinco da escala. Observando a média dos itens na Tabela 3, pode-se verificar quais são os itens desequili- 
Tabela 5. Análise fatorial exploratória dos 50 itens: fatores e cargas.

\begin{tabular}{|c|c|c|c|c|c|c|c|}
\hline Itens/Fatores & 1 & 2 & 3 & 4 & 5 & 6 & 7 \\
\hline & Int-Ext & ANE & Mut-FO & Est & FObj-FRH & RACIONAL & AEC \\
\hline Gosta de fazer várias coisas & $-0,25$ & 0,29 & 0,06 & 0,10 & 0,02 & 0,01 & 0,02 \\
\hline Sintonizado com várias emoções & 0,02 & 0,08 & 0,67 & $-0,05$ & $-0,06$ & 0,11 & $-0,08$ \\
\hline Persistente & $-0,09$ & 0,09 & 0,07 & 0,03 & 0,26 & 0,32 & $-0,29$ \\
\hline Estável & 0,04 & $-0,02$ & $-0,09$ & 0,46 & $-0,05$ & 0,33 & $-0,03$ \\
\hline Prático & $-0,08$ & 0,15 & $-0,10$ & 0,29 & 0,09 & 0,23 & $-0,08$ \\
\hline Falador & $-0,63$ & 0,18 & 0,09 & $-0,09$ & $-0,20$ & 0,03 & $-0,12$ \\
\hline Reservado & 0,62 & 0,07 & 0,09 & 0,02 & 0,01 & 0,23 & $-0,01$ \\
\hline Inventivo & 0,12 & 0,68 & $-0,02$ & $-0,04$ & 0,04 & $-0,02$ & $-0,34$ \\
\hline Comunicativo & $-0,62$ & 0,27 & 0,01 & 0,02 & 0,11 & 0,02 & $-0,01$ \\
\hline Influenciado pelas emoções & 0,06 & 0,05 & 0,70 & $-0,15$ & $-0,06$ & 0,02 & 0,05 \\
\hline Caloroso & $-0,20$ & 0,04 & 0,39 & 0,07 & $-0,04$ & 0,13 & $-0,08$ \\
\hline Calmo & 0,46 & $-0,04$ & 0,00 & 0,72 & $-0,06$ & $-0,05$ & $-0,05$ \\
\hline Racional & 0,05 & $-0,07$ & $-0,02$ & 0,09 & 0,13 & 0,54 & $-0,02$ \\
\hline Gosta de coisas já comprovadas & 0,06 & $-0,01$ & $-0,07$ & $-0,13$ & 0,11 & 0,41 & 0,13 \\
\hline Desapegado & 0,05 & 0,11 & $-0,26$ & $-0,08$ & $-0,05$ & 0,21 & $-0,07$ \\
\hline Focado & 0,05 & 0,02 & 0,05 & $-0,01$ & 0,48 & 0,35 & $-0,19$ \\
\hline Prestativo & $-0,07$ & 0,28 & $-0,04$ & 0,09 & 0,47 & 0,04 & 0,07 \\
\hline Não emotivo & 0,04 & 0,13 & $-0,59$ & 0,06 & $-0,17$ & 0,27 & $-0,01$ \\
\hline Introvertido & 0,20 & 0,01 & $-0,07$ & 0,02 & $-0,28$ & 0,25 & 0,15 \\
\hline Desembaraçado & $-0,11$ & 0,15 & 0,07 & 0,04 & $-0,05$ & 0,29 & $-0,27$ \\
\hline Criativo & $-0,04$ & 0,76 & $-0,06$ & $-0,02$ & 0,11 & $-0,04$ & $-0,24$ \\
\hline Curioso & $-0,23$ & 0,35 & 0,10 & $-0,09$ & $-0,09$ & 0,14 & 0,05 \\
\hline Quieto & 0,75 & 0,04 & $-0,05$ & 0,24 & 0,17 & $-0,03$ & 0,05 \\
\hline Não gosta de mostrar muito afeto & 0,21 & 0,23 & $-0,42$ & $-0,16$ & $-0,04$ & 0,21 & 0,09 \\
\hline Gentil & 0,00 & 0,16 & 0,13 & 0,34 & 0,27 & $-0,02$ & 0,28 \\
\hline Começa as coisas sem a obrigação de terminá-las & 0,03 & 0,25 & 0,05 & 0,04 & $-0,48$ & 0,03 & 0,10 \\
\hline Equilibrado & 0,09 & 0,04 & $-0,05$ & 0,46 & 0,13 & 0,25 & 0,11 \\
\hline Tradicional & $-0,01$ & 0,00 & 0,02 & 0,24 & 0,04 & 0,08 & 0,45 \\
\hline Extrovertido & $-0,53$ & 0,24 & 0,15 & 0,05 & 0,03 & 0,00 & 0,03 \\
\hline Cooperativo & $-0,06$ & 0,26 & 0,13 & 0,12 & 0,40 & 0,04 & 0,12 \\
\hline Humor variável & 0,05 & 0,29 & 0,11 & $-0,27$ & $-0,03$ & $-0,01$ & 0,30 \\
\hline Afetivo & $-0,07$ & $-0,04$ & 0,66 & 0,24 & 0,05 & 0,06 & $-0,07$ \\
\hline Empreendedor & $-0,02$ & 0,20 & 0,18 & 0,09 & 0,02 & 0,36 & $-0,14$ \\
\hline Age com os sentimentos & $-0,01$ & 0,07 & 0,72 & 0,00 & $-0,07$ & $-0,03$ & 0,22 \\
\hline Não gosta de especular & 0,10 & 0,03 & 0,12 & 0,04 & $-0,03$ & 0,20 & 0,01 \\
\hline Original & $-0,25$ & 0,28 & $-0,03$ & 0,06 & 0,14 & 0,05 & 0,03 \\
\hline Bom de papo & $-0,51$ & 0,27 & $-0,02$ & 0,10 & 0,12 & $-0,03$ & $-0,02$ \\
\hline Prefere agir ao invés de se relacionar & $-0,09$ & 0,16 & $-0,18$ & $-0,08$ & $-0,07$ & 0,21 & 0,21 \\
\hline Autodisciplinado & 0,19 & 0,04 & 0,05 & 0,16 & 0,45 & 0,16 & 0,09 \\
\hline Tranquilo & 0,35 & 0,04 & 0,04 & 0,72 & 0,03 & 0,03 & 0,03 \\
\hline Decidido & $-0,17$ & 0,07 & $-0,02$ & 0,16 & 0,29 & 0,26 & $-0,02$ \\
\hline Constante & $-0,14$ & $-0,07$ & $-0,02$ & 0,33 & $-0,01$ & 0,47 & 0,08 \\
\hline Gosta de coisas racionais & $-0,05$ & $-0,15$ & 0,00 & $-0,04$ & 0,09 & 0,77 & 0,10 \\
\hline Alternativo & 0,02 & 0,33 & 0,11 & 0,06 & 0,00 & 0,10 & 0,03 \\
\hline Conservador & 0,01 & 0,06 & $-0,03$ & 0,03 & 0,13 & 0,23 & 0,37 \\
\hline Emocional & 0,04 & $-0,01$ & 0,76 & $-0,15$ & 0,08 & $-0,05$ & 0,23 \\
\hline Gosta de cumprir metas & 0,07 & $-0,03$ & 0,05 & $-0,09$ & 0,54 & 0,24 & $-0,04$ \\
\hline Prefere experimentar a ter metas definidas & $-0,01$ & 0,19 & 0,16 & 0,12 & $-0,22$ & 0,08 & 0,10 \\
\hline Discreto & 0,58 & 0,07 & 0,01 & 0,05 & 0,18 & 0,15 & $-0,03$ \\
\hline Fechado & 0,68 & $-0,02$ & 0,05 & $-0,05$ & $-0,11$ & 0,18 & $-0,04$ \\
\hline
\end{tabular}

Int-Ext: Introversão-Extroversão; ANE: Abertura a Novas Experiências; Mut-FO: Mutabilidade-Foco no Objeto, Est: Estabilidade; FObj-FRH: Foco no Objetivo-Foco em Relações Humanas; AEC: Abertura a Experiências Consolidadas. 
brados que pendem para o grupo dos pontos um e dois, e quais são os itens desequilibrados que pendem para o grupo dos pontos quatro e cinco. Os primeiros podem ser definidos como difíceis, pois há maior probabilidade de que a característica presente em cada um desses itens seja relatada como pouco presente por boa parte dos participantes. Os últimos, por consequência, podem ser definidos como itens fáceis, pois há maior probabilidade de que a característica presente em cada um desses itens seja relatada como muito presente por boa parte dos participantes. Através dessa análise, pôde-se definir a presença de quatro itens difíceis: reservado, não emotivo, não gosta de mostrar muito afeto e fechado. O número de itens desequilibrados fáceis é 18 e representa a grande maioria dos itens do ICP.

Finalizando, poder-se-ia argumentar que a estratégia da análise fatorial exploratória foi conduzida inadequadamente pelo seguinte motivo: ao realizar análises fatoriais exploratórias de segmentos (grupos) de itens, enquanto primeira estratégia para seleção de itens, obteve-se possivelmente uma restrição na análise da estrutura das variâncias e covariâncias dos 50 itens do instrumento. Tal argumento é pertinente do ponto de vista eminentemente exploratório. Por sua vez, é importante salientar que a elaboração do instrumento em questão foi gerada fundamentalmente por um motivo teórico, de necessidade de criação de um instrumento capaz de aferir separadamente as polaridades opostas do big five, assim como não introduzir significativo viés valorativo. Itens capazes de mensurar o maior número possível de polaridades opostas foram selecionados. Nesse sentido, a escolha pelos itens foi dominada pela necessidade imposta pelo propósito teórico, sem a desconsideração, evidente, do plano empírico. Apesar da valorização da teoria, deve-se ressaltar que a escolha pela seleção de itens agrupados mostrou-se consistente empiricamente. Ao realizar uma análise fatorial exploratória de todos os 50 itens, sem segmentá-los, foi identificada uma solução de sete fatores $\left(\chi^{2}=345,39, \mathrm{gl}=163, \mathrm{CF}=0,99, \mathrm{RMSEA}=0,04, \mathrm{SRMR}=0,02\right)$, capaz de explicar $46,03 \%$ da variabilidade dos itens (Tabela 4). A Tabela 4 mostra os autovalores obtidos da matriz policórica analisada, enquanto a Tabela 5 apresenta os fatores e as cargas fatoriais dos itens nos fatores

218 obtidos.
O fator 1 (Int-Ext) é uma aglutinação das polaridades inversas introversão e extroversão. O fator 2 (ANE) é a polaridade abertura a novas experiências. $O$ fator 3 (Mut-FO) aglutina a polaridade mutabilidade e alguns itens da polaridade foco no objeto. O fator 4 (Est) é a polaridade estabilidade. $\mathrm{O}$ fator 5 (FObj-FRH) aglutina as polaridades foco no objetivo e alguns itens de foco em relações humanas. $O$ fator 6 é um traço específico de racionalidade ou pensamento racional. 0 fator 7 indica a polaridade de abertura a experiências consolidadas, e seus itens marcadores possuem cargas fatoriais mais fracas.

Em síntese, boa parte dos fatores encontrados e seus respectivos itens da AFE de 50 itens são consonantes com os resultados deste estudo, provenientes da análise inicial de grupos de itens. A escolha pelos 27 itens, ao contrário de restringir a análise, ampliou o número de polaridades opostas identificadas, permitindo a mensuração de oito polos do big five. É certo, também, que algumas dessas polaridades possuem baixo alfa e carecem de maior número de itens, o que implica a necessidade de melhorias do instrumento.

\section{Considerações Finais}

Este artigo concentrou sua análise na estrutura fatorial do ICP e no grau de dificuldade dos itens do instrumento. As evidências obtidas sustentam dizer que o ICP é um instrumento promissor para a mensuração não valorativa e separada das polaridades das dimensões do big five. $\mathrm{O}$ instrumento mensura oito das dez polaridades do modelo.

Não obstante, as evidências também indicam que o instrumento deve ser aprimorado. Quatro polaridades medidas pelo ICP não são confiáveis (baixo alfa) e duas polaridades pretendidas não foram identificadas: abertura a experiências consolidadas e foco em várias atividades.

Decerto, a presente investigação é um estudo exploratório inicial a respeito da validade de construto do ICP, de modo que desafios futuros necessitam ser trabalhados. Um desses desafios envolve compreender se há invariância da estrutura fatorial do ICP, em termos dos estudantes do ensino fundamental e ensino médio. Na medida em que boa parte dos itens do instrumento 
é composta por adjetivos, é de se esperar que a série e a idade dos estudantes influenciem sua compreensão das palavras, o que por sua vez pode influenciar o padrão de respostas aos itens do instrumento.

Outro desafio envolve compreender se há diferenças entre distintos grupos etários, série escolar, ou gênero, no que tange aos traços medidos.

Um terceiro desafio envolve a análise da validade convergente e divergente, assim como a análise da validade de critério, de modo a compreender a relação do ICP com os instrumentos validados para a população brasileira que medem as dimensões do big five.

Um quarto desafio envolve a própria qualidade do ICP.É necessário aumentar o número de itens capazes de mensurar cada traço do instrumento. Poder-se-ia definir que o ideal seria que pelo menos oito a dez itens fossem capazes de mensurar adequadamente cada um dos oito traços já mensurados. Além do número de itens, é relevante que cada traço seja mensurado por itens classificados neste trabalho como equilibrados, desequilibrados fáceis e desequilibrados difíceis. Não é ideal que um traço seja mensurado quase exclusivamente por itens equilibrados ou por itens desequilibrados fáceis, por exemplo.

Um quinto desafio envolve construir itens capazes de mensurar os dois traços não identificados pelos itens atuais do ICP: abertura a experiências consolidadas e foco em várias atividades.

Um sexto desafio envolve observar a validade preditiva do ICP. Deve-se investigar se a identificação dos dez traços traz contribuições para a predição do desempenho acadêmico e profissional, entre outros campos.

Um sétimo desafio envolve investigar se há relação intensa entre a habilidade de compreensão verbal e o padrão de respostas dos participantes no ICP. Deve-se verificar se o ICP depende fortemente da compreensão verbal, a ponto de indicar algum viés.

Implicações e contribuições do ICP podem ser apontadas. A primeira delas envolve a problematização referente à própria construção dos termos e itens que mensuram o big five. A literatura do big five apresenta termos e itens que privilegiam a sobrevalorização de uma polaridade, em detrimento da outra polaridade de cada uma das dimensões do modelo, conforme já argumentado. O ICP traz a perspectiva de uma nova abordagem e uma nova agenda de investigações.

Uma segunda implicação envolve a compreensão de como traços valorados de forma negativa pela literatura do big five, como o neuroticismo, se relacionam a determinados desempenhos particulares a partir da perspectiva do ICP. O ICP remodela o termo neuroticismo e define-o como mutabilidade, ou seja, uma influência significativa do fluxo emocional sobre o indivíduo, que evidentemente pode ser positiva ou negativa, dependendo da intensidade e do contexto. É de se esperar que algumas áreas cotidianas ou profissionais sejam relacionadas positivamente pela influência do traço mutabilidade, como por exemplo, a capacidade de representação no teatro.

Uma terceira implicação diz respeito à importância da teoria. Deve-se atentar para o fato de que, dos 50 itens iniciais do ICP, apenas 27 foram mantidos. Sem um direcionamento teórico a respeito dos traços, muito provavelmente os oito fatores encontrados não teriam sido adequadamente identificados neste trabalho. Sem uma teoria que possa servir de ponto de partida, métodos fatoriais de tratamento de dados mostram-se muitas vezes insuficientes. Por outro lado, a articulação entre uma boa teoria e métodos fatoriais de análise de dados tem-se mostrado um casamento frutífero.

\section{Referências}

Baker, S. R., Victor, J. B., Chambers, A. L., \& Halverson, C. F. (2004). Adolescent personality: a five-factor model construct validation. Assessment, 11 (4), 303-315.

Barbaranelli, C., \& Caprara, G. V. (2002). Studies of the big five questionnaire. In B. Raad \& M. Perugini (Orgs.), Big five assessment (pp.109-128). Seattle: Hogrefe \& Huber Publishers.

Bentler, P. M. (1990). Comparative fit indexes in structural models. Psychological Bulletin, 107, 238-246.

Browne, M. W., \& Cudeck, R. (1993). Alternative ways of assessing model fit. In K. A. Bollen \& J. S. Long (Orgs.), Testing structuralequation models (pp. 136-162). Newbury Park, CA: Sage.

Byrne, B. M. (2001). Structural equation modeling with AMOS: basic concepts, applications, and programming. Mahwah, NJ: Lawrence Erlbaum Associates.

Costa. Jr., R. T., \& McCrae, R. R. (1992). NEO-PI-R professional manual: revised NEO Personality Inventory (NEO-PI-R) and NEO-Five Factor Inventory (NEO-FFI). Odessa, FL: Psychological Assessment Resources. 
Costa Jr., R. T., McCrae, R. R., \& Jónsson, F. H. (2002).Validity and utility of the revised NEO personality inventory examples from Europe In B. Raad \& M. Perugini (Orgs.), Big five assessment (pp.61-78). Seattle: Hogrefe \& Huber Publishers.

Goldberg, L. R. (1990). An alternative "description of personality: the big-five factor structure. Journal of Personality and Social Psychology, 59 (6), 1216-1229.

Goldberg, L. R. (1992). The development of markers for the big-five factor structure. Psychological Assessment, 4 (1), 26-42.

Hendriks, A. A. J., Hofstee, W. K. B., \& Raad, B. (2002). The five-factor personality inventory: assessing the big five by means of brief and concrete statements. In B. Raad \& M. Perugini (Orgs.), Big five assessment (pp.79-108). Seattle: Hogrefe \& Huber Publishers.

Hu, L., \& Bentler, P. M. (1999). Cutoff criteria for fit indexes in covariance structure analysis: conventional criteria versus new alternatives. Structural Equation Modeling, 6 (1), 1-55.

Hutz, C. S., \& Nunes, C. H. (2001). Escala fatorial de ajustamento emocional/neuroticismo: EFN. São Paulo: Casa do Psicólogo.

Hutz, C. S., Nunes, C. H. S. S., Silveira, A. D., Serra, J., Anton, M., \& Wieczorek, L. S. (1998). O desenvolvimento de marcadores para a avaliação da personalidade no modelo dos cinco grandes fatores. Psicologia: Reflexão e Crítica, 11 (2), 395-409.

John, O.P. (1990). The "big five" factor taxonomy: dimensions of personality in the natural languages and in questionnaires. In L. A. Pervin (Org.), Handbook of personality: theory and research (pp.66-100). New York: Guilford Press.

McCrae. R. R., \& John, O. P. (1992). An introduction to the five-factor model and its applications. Journal of Personality, 60 (2), 175-215.

Mervielde, I., \& Fruyt, F. (2002). Assessing children's traits with the hierarchical personality inventory for children. In B. Raad \& M. Perugini (Orgs.), Big five assessment (pp.129-146). Seattle: Hogrefe \& Huber Publishers.

Nunes, C. H. S. S. (2000). A construção de um instrumento de medida para o fator neuroticismo/estabilidade emocional dentro do modelo de personalidade dos cincograndesfatores.
Dissertação de mestrado não-publicada, Programa de Pós-Graduação, Universidade Federal do Rio Grande do Sul, Porto Alegre.

Nunes, C. H. S. S. (2007). Lançamento da escala fatorial de extroversão (EFEx) e escala fatorial de socialização (EFS). Avaliação Psicológica, 6 (1), 103-106.

Nunes, C. H. S. S., \& Hutz, C. S. (2007). Construção e validação da escala fatorial de socialização no modelo dos cinco grandes fatores de personalidade. Psicologia, Reflexão e Crítica, 20 (1), 20-25.

Paunonen, S. V., \& Ashton, M. C. (2002). The nonverbal assessment of personality: the NPQ and the FF-NPQ. In B. Raad \& M. Perugini (Orgs.), Big five assessment (pp.171-194). Seattle: Hogrefe \& Huber Publishers.

Perugini, M., \& Di Blas, L. (2002). The big five marker scales (BFMS) and the italian AB5C taxonomy: analyses from an etic-emic perspective. In B. Raad \& M. Perugini (Orgs.), Big five assessment (pp.281-304). Seattle: Hogrefe \& Huber Publishers.

Pinheiro, C. A. R., Gomes, C. M. A., \& Braga, A. G. (2009). Construção e validação do inventário dos adjetivos de personalidade - 50 (IAP-50). Anais do IV Congresso Brasileiro de Avaliação Psicológica, VCongresso da Associação Brasileira de Rorschach e Métodos Projetivos, XIV Conferência Internacional de Avaliação Psicológica: formas e contextos. Campinas: Instituto Brasileiro de Avaliação Psicológicas.

Rabelo, I. S., Leme, I. F. A. S., \& Rossetti, M. O., Pacanaro, S. V., Guntert, I. B., \& Barrionuevo, V. L. (2009). Aplicação das escalas de personalidade EFS e EFEx no contexto organizacional. Avaliação Psicológica, 8 (1), 109-117.

Schmit, M. J., Kihm, J. A., \& Robie, C. (2002). The global personality inventory (GPI). In B. Raad \& M. Perugini (Orgs.), Big five assessment (pp.195-236). Seattle: Hogrefe \& Huber Publishers.

Tsaousis, I. (2002). The traits personality questionnaire (TPQue). In B. Raad \& M. Perugini (Orgs.), Big five assessment (pp.237-260). Seattle: Hogrefe \& Huber Publishers.

Recebido em: 6/5/2010

Versão final reapresentada em: 30/8/2011

Aprovado em: 19/9/2011 